\title{
Membrane progestin receptors: beyond the controversy, can we move forward?
}

\author{
Amel Salhi, Julie Lemale, Nicolas Paris, May \\ Bloch-Faure and Gilles Crambert* \\ UPMC Univ Paris 6/INSERM/CNRS, Centre de Recherche \\ des Cordeliers, UMRS 872 Equipe 3 Laboratoire de \\ Génomique, Physiologie et Physiopathologie Rénales \\ ERL 7226, F-75006, Paris, France \\ * Corresponding author \\ e-mail: gilles.crambert@crc.jussieu.fr
}

\begin{abstract}
Steroids are well-known mediators of many different physiological functions. Their best characterized mechanism of action involves interaction with well-defined nuclear receptors and regulation of gene transcription. However, rapid effects of steroids have been reported which are incompatible with their classical long-term/slow effects. Although the concept of membrane-bound receptors for steroids which can transduce their rapid effects has been proposed many years ago, it is only recently that such proteins have been identified and characterized. In this review, we will discuss recent data regarding the rapid action of progesterone mediated by newly characterized membrane-bound receptors belonging to the progestin and adiponectin receptor family.
\end{abstract}

Keywords: membrane-bound receptors; non-genomic action; progesterone; steroid.

\section{Introduction}

The way in which steroids act is mainly described as a slow process involving gene transcription. This mechanism, known as the "genomic" pathway, was postulated more than 60 years ago. During the past decades, this mechanism has been investigated and particularly well described. It involves (i) the transport of steroid into the cell (either passively or using putative carriers); (ii) the binding of the hormone to receptors trapped in the cytoplasm; (iii) the dimerization and translocation of the ligand-receptor complex to the nucleus; and (iv) the binding to specific nucleotide motifs and activation of transcription (Figure 1). The receptors mediating this "genomic" action belong to the steroid nuclear receptor superfamily.

The main characteristics of this process are therefore a delay of many hours between hormone treatment (release/ administration?) and related biological effects, as well as the involvement of a nuclear receptor. However, steroids have also been reported to have biological effects within minutes, as well as effects in cells or tissues devoid of their specific nuclear receptors. These rapid and non-classical effects were originally described for progesterone (1) and then reported for all major classes of steroids $(2,3)$, and particularly for progesterone in various physiological contexts such as oocyte maturation (4), induction of acrosomal reaction in sperm [for review see Ref. (5)], and activation of calcium uptake by renal proximal tubules $(6,7)$. Many explanations have been put forward to support these observations [for review see Ref. (8) and Figure 1]: (i) the disturbance of the membrane fluidity by insertion of a lipophilic molecule in the lipid bilayer which can then influence activity of transporters, channels etc.; (ii) the action of nuclear receptors at the plasma membrane; (iii) the action of nuclear receptor coregulators released upon receptor activation by the hormone; and (iv) involvement of a membrane-bound receptor, unrelated to the classical nuclear receptor. In view of the plethoric physiological and pathophysiological effects of steroids, the discovery of such receptors and the characterization of their signaling pathways could have considerable importance, for instance to envisage new therapeutic strategies.

In this review, we will discuss one example, namely the hormone progesterone, and the recent and controversial identification of the so-called membrane progestin receptors (mPRs). The purpose of this paper is to contribute to the debate initiated by Fernandes et al. (9), who proposed to share different points of view present in this field.

\section{Original observations and subsequent work by the Peter Thomas group}

During the late 1980s and early 1990s, the Peter Thomas group at the Marine Science Institute of the University of Texas (Austin, TX, USA) identified a novel progestin (17,20$\beta, 21$-trihydroxy-4-pregnen-3-one or 20ß-S) as the oocyte maturation-inducing steroid in the teleost species $(10,11)$. The binding of this novel progestin was shown to occur at the plasma membrane of oocytes with rapid dissociation and association rates (12). To identify the putative receptors involved in the binding of this steroid, this group developed an original and elegant multisteps procedure involving partial protein purification steps to serve as an antigen to produce monoclonal antibodies, a novel receptor-capture assay to isolate the antibodies recognizing the putative progestin receptors, and the screening of a library expressing proteins from sea trout ovaries with this selected antibody to isolate the putative membrane progestin receptor cDNA. This meth- 


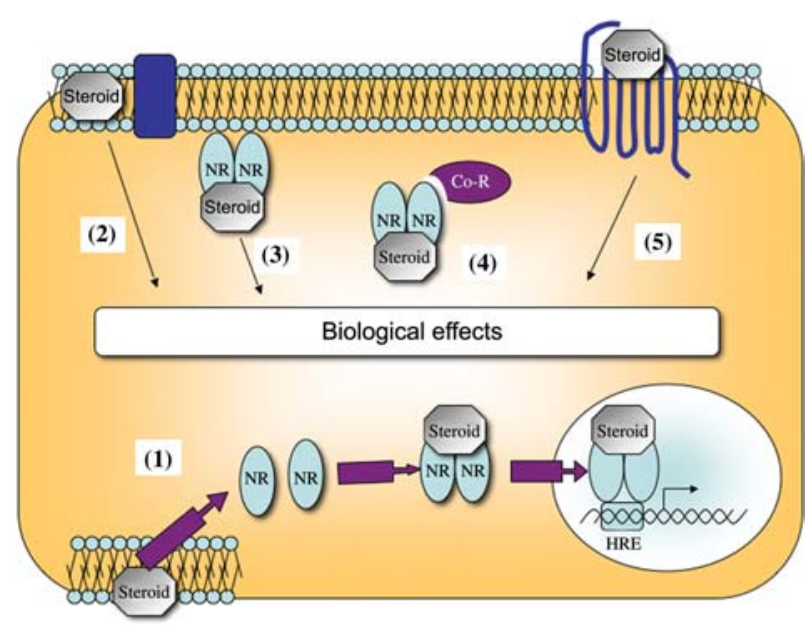

Figure 1 Schematic representation of classical and non-classical steroid actions.

Steroids elicit classical, genomic effects (1) by binding to specific nuclear receptors (NRs) which then dimerize and are translocated into the nucleus. The steroid-bound receptor recruits different coregulator forming a transcription complex and then binds to a hormone response element (HRE) and triggers gene transcription. Steroids can also trigger rapid, non-classical effects in different ways: disturbance of the lipid bilayer fluidity and modulation of membrane protein activity (2), binding to a pool of nuclear receptors present at the cell surface (3), release of nuclear receptor co-regulators (Co-R) having their own activity (4), and binding to membrane-bound receptors (5).

od successfully led to the identification of a new class of protein, called mPRs $(13,14)$. Research for orthologs revealed that mPRs are well conserved in different species including mammals and particularly humans. In vertebrates, there are at least three classes of receptors $(\alpha, \beta$, and $\gamma)$ which are differentially distributed and share $50-60 \%$ of homology at the protein level. The $\alpha$ receptor is mainly found in sex organs and to a lesser extent in the bladder, the adrenal glands, and the kidney. The $\beta$ form is only neuronal and the $\gamma$ form is found in epithelial tissues. It is noteworthy that the kidney is, so far, the only organ to express two forms, the $\alpha$ and the $\gamma$ receptors. A more detailed study regarding this non-reproductive organ has shown specific localization of these isoforms along the nephron without any incidence of gender on their level of expression (15). According to their sequences, the three isoforms, $\mathrm{mPR} \alpha, \beta$, and $\gamma$ belong to a family of protein comprising adiponectin receptors 1 and 2, osmotin receptor, and RKTG (Raf Kinase Trapping in Golgi also referred to as PAQR3) (16-18). This new family has been named Progestin- and AdipoQ-Receptor family (PAQR) and displays not only sequence homology but also a similar seven-transmembrane domains structure and a common ancestor (19). Proteins from plants have also been identified as members of this PAQR family (20). As they display the predicted seven-transmembrane domains topology, they have been named accordingly (HHP, HeptaHelical Protein 1-5). Experimental evidence $(16,18)$ has shown that although adiponectin receptors and RKTG/ PAQR3 display predicted topology including seven trans- membrane domains, they exhibit an inverted topology when compared with G-protein-coupled receptors (GPCRs).

\section{Membrane progestin receptors function as GPCRs}

Although some members of the PAQR family are unrelated to GPCRs, observations reported by the Peter Thomas group indicate that $\mathrm{mPRs}$ display functional characteristics of this class of receptors. First of all, in contrast to adiponectin receptors and RKTG, the topology of $\mathrm{mPR}$, as described by the Peter Thomas group, is that of a GPCR. In the original description $(13,14)$, the topology of mPR, with seven transmembrane domains and an extracellular N-terminus part, was only extrapolated from in silico sequence analysis. Later, they showed that an antibody directed against the N-terminal part of sea trout $\mathrm{mPR} \alpha$ recognized its target in non-permeabilized cells, thereby establishing the extracellular localization of the N-terminal tail $(21,22)$. It should be underscored that the presence of seven transmembrane domains has never been experimentally proven: neither for $\mathrm{mPR}$ nor for any PAQR members. The main evidence of the Peter Thomas group suggesting that $\mathrm{mPR}$ are GPCRs was first obtained in cells stably transfected with sea trout $\mathrm{mPR} \alpha$, where addition of either progesterone or $20 \beta-S$ induced a pertussis toxinsensitive decrease of cAMP, suggesting an inhibition of the adenylyl cyclases by a $\mathrm{G}_{0}$ or a $\mathrm{G}_{\mathrm{i}}$ protein (13). Later, using native tissue, they demonstrated an interaction between $\mathrm{mPR} \alpha$ or $\beta$ with the inhibitory $\mathrm{G}_{\mathrm{i}}$ protein in human myometrial cells (23). More recently, Tubbs and Thomas $(24,25)$ showed that progestin-induced sperm hypermotility was related to $\mathrm{mPR} \alpha$ stimulation followed by activation of a stimulatory olfactory $G$ protein $\left(G_{\text {olf }}\right)$ which induces an increase in intracellular cAMP. Thus, depending on tissues or cells, mPR could be associated with either an inhibitory or a stimulatory $\mathrm{G}$ protein inducing opposite effects on cAMP cellular content. This observation is intriguing as many tissues or cells expressed both $\mathrm{Gi}$ and $\mathrm{G}_{\text {olf }}$ proteins. The question is therefore how mPRs "choose" which G protein they should associate with. The MAP kinase pathway is also triggered by activation of $\mathrm{mPR}$ proteins (13) but the link with GPCR activity has not been established.

\section{Subcellular localization of mPR}

Supporting this GPCR function, the Peter Thomas group consistently observed the expression of mPR (irrespective of the isoform or the species) at the plasma membrane by using different techniques $(13,21,22)$. However, these authors also reported the co-localization of $\mathrm{mPR} \alpha$ and $\beta$ with the nuclear progesterone receptor in internal vesicles (23). This point and particularly the apparent paradoxical finding that $\mathrm{mPR}$ is present both at the plasma membrane and in internal compartments is not discussed further by the authors.

Zhu and collaborators (26) have reported that cellular localization of $\mathrm{mPR}$ is fused to a large fluorescent protein (YFP) either on $\mathrm{N}$ - or $\mathrm{C}$-terminal positions. The authors concluded that mPR transfection is "toxic" for the cells and that the presence of epitope tags at the $\mathrm{N}$-terminus extremity is responsible for retention of $\mathrm{mPR}$ into intracellular com- 
partment, whereas C-terminus tag should permit plasma membrane expression. However, even though the retention of the N-terminus-tagged construction is clear, the presence of the C-terminus-YFP mPR at the plasma membrane is not obvious despite the authors' assertions.

\section{mPR are specific receptors for progesterone and its metabolites}

Pharmacological characteristics of $\mathrm{mPR}$ have been well described in different reports from the Peter Thomas group (13, 27, 28). By overexpressing recombinant mPR in Escherichia coli, binding experiments of $\left(\mathrm{H}^{3}\right)$ progesterone showed the presence of a single class of receptors with a high affinity $\left(K_{\mathrm{d}}\right.$ around $\left.30 \mathrm{~nm}\right)$ whatever the isoforms and the species taken into account. The dissociation and association rate constants are both rapid (2-5 min). The specificity of mPR was shown to be restricted to progesterone or its metabolites (17 $\alpha$-hydroxyprogesterone and $20 \beta$-hydroxyprogesterone) (13, $27,28)$. Among known nuclear progesterone- or estrogenor androgen-receptor antagonists tested, none of them has any effect on progesterone binding to $\mathrm{mPR}$.

In summary, the important work done by the Peter Thomas group has identified a protein family localized at the plasma membrane with a seven-transmembrane domains topology, coupled to $G$ proteins and specific for progesterone. These conclusions are supported by approximately 15 articles published during the past 5 years, exploring $\mathrm{mPR}$ in different species, tissues, and cells. Unfortunately, when mPRs have been investigated by other groups, many important observations (topology, coupling with $G$ proteins, plasma membrane localization, and even ligand specificity) have been entirely or partially refuted, thereby leading to significant confusion in this research field. Peter Thomas recently answered some of these contradictions by deploring that "despite the mounting evidence that mPRs are functional membrane progestin receptors", researchers "have continued to dispute these conclusions based on negative findings...' (25). As the best criterion for unambiguously accepting a new concept is the confirmation of this concept by other independent research groups, negative results are not trivial and could be significant. In the following section, we endeavor to analyze these "negative findings" and see whether they are relevant or not, and, if so, what they could teach us.

\section{mPRs: the dissonant points of view}

Based on the exciting discovery by Zhu and colleagues in $2003(13,14)$ of a new family of membrane-bound progesterone receptors, many groups have undertaken their own research work with the hope to identify new candidates that would explain the rapid and non-genomic effects of progesterone in their physiological models. Three main points have been particularly debated: (i) the ligand-specificity of these receptors; (ii) their localization at the plasma membrane; and (iii) the assertion that mPRs function as GPCRs.

\section{Does progesterone activate mPRs?}

The rationale for this question came from the study by Krietsch et al. (29) who suggested that mPRs were not able to bind progesterone. This group cloned human $\mathrm{mPR} \alpha, \beta$, and $\gamma$ and sea trout and Fugu $\operatorname{mPR} \alpha$, all of them fused with HA-epitope, and stably expressed in HEK293 or MDA-MB231 cell lines. Under all these conditions, progesterone incubation did not lead to any change in intracellular cAMP content nor ERK or p38 phosphorylation. Using crude membrane or microsomal preparations from these stable cell lines, these authors were not able to confirm progesterone binding related to the expression of one or another mPR. It is possible, however, that the strategy used by Krietsch et al. does not allow one to measure these parameters as MDAMB-231 and HEK293 express endogenous progesterone receptors (mPR or others). In our study (30), focusing on mPRs in renal epithelium, we observed neither increased phosphorylation of ERK nor increased intracellular $\mathrm{Ca}^{2+}$ after progesterone treatment on tubules expressing endogenous $m P R \alpha$ and $m P R \gamma$. However, in this study we did not conclude that mPRs were not progesterone receptors as no binding experiments were performed. Two recent publications, from 2008 and 2009, suggest that mPRs are indeed progesterone receptors. Using a mammalian cell line (CHO) expressing ovine $\mathrm{mPR} \alpha$, Ashley et al. (31) have shown a specific binding of progesterone when compared with untransfected cells. This ovine $\mathrm{mPR} \alpha$ exhibits a slightly different affinity for progesterone than that reported for sea trout or human mPR but it exhibits a similar ligand-specificity profile. In addition to these studies, the group of T. Lyons used a very elegant strategy using yeast (Saccharomyces cerevisiae) as an expression system for mammalian PAQR proteins. Indeed, this organism has been shown to express a PAQR protein, the osmotin receptor, and Kupchak et al. (32) previously described the transduction pathway elicited by its activation leading to downregulation of a gene named FET3. This group has shown that heterologous expression of adiponectin receptor, upon adiponectin stimulation, also leads to downregulation of FET3. They have used the same strategy to investigate the ligand-dependency of $\mathrm{mPR} \alpha, \beta$, and $\gamma$ and have shown that these proteins, indeed, are stimulated by progesterone $(33,34)$. They have also used this strategy to demonstrate the activation of the orphan receptors PAQR6 and PAQR9 by progesterone (34). These two PAQR members are now referred to as $\mathrm{mPR} \delta$ and $\mathrm{mPR} \varepsilon$. Therefore, convergent conclusions, from different groups using different strategies, indeed confirm that mPRs are receptors for progesterone. However, the transduction pathways triggered by their stimulation is probably tissue- or cell-dependent.

\section{Plasma membrane or not plasma membrane...?}

The localization of mPR has probably been one of the most disputed aspects of its characterization. In 2005 and 2006, three independent reports described mPRs in the endoplasmic reticulum (ER) but not at the plasma membrane $(29,35$, 36). In these studies, the heterologous expression of HA-, V5-, GFP-epitope-tagged mPR ( $\alpha, \beta$, or $\gamma$ isoforms from 
different species) in different cell lines (CHO, Cos-7, HEK 293, MDA-MB-231, small and large luteal cells) led to the conclusion that $\mathrm{mPRs}$ are retained in the ER, as proven either by co-localization with ER markers or by the absence of co-localization with plasma membrane labeling. Moreover, Krietsch et al. (29) showed that immunocytochemical experiments failed to reveal any signal on non-permeabilized cells. It is unlikely that mPR retention in ER is an artifact as a result of the presence of tag sequences, as proposed by Zhu et al. (26), because in these studies wild type mPRs were also transfected and exhibited the same cellular localization as the tagged constructions (29). ER retention induced by overexpression of exogenous proteins does not seem to be a good explanation either, as endogenous $\mathrm{mPR} \alpha$ isoforms present in MDA-MB-231 (derived from breast cancer adenocarcinoma) and Ishikawa (derived from human endometrial adenocarcinoma) cells exhibit a reticulum specific localization (29). More recently, we investigated the putative role of mPRs on the rapid effect of progesterone on renal tubules (30). Using our own antibodies recognizing murine $\mathrm{mPR} \alpha$ and $\mathrm{mPR} \gamma$, we showed by differential centrifugation on iodixanol density gradients followed by Western blot analysis that in native renal tissue, mPRs were not present in the same fraction as markers of the plasma membrane $(\mathrm{Na}, \mathrm{K}-$ ATPase and $\mathrm{Na}, \mathrm{H}$-exchanger-3). By using heterologous expression of murine $\mathrm{mPR} \alpha$, we confirmed its presence in ER, independently of the nature or the position of the tag and the cell line used for transfection (Figure 2). We also established the presence of an ER retention/retrieval motif $(-\mathrm{KXX})$ at the C-terminal extremity of $\mathrm{mPR} \alpha$. It is noteworthy that the removal of this motif was not sufficient to yield cell surface expression but led to accumulation into a Golgi-like structure. As shown in Figure 3, the role of this motif is confirmed in mouse mPR $\gamma$, as replacement of the last three amino acids from lysine 328 by three alanines changes the localization of the receptor.

With the exception of the Peter Thomas group, results obtained with homemade antibodies, there seems to be a con-

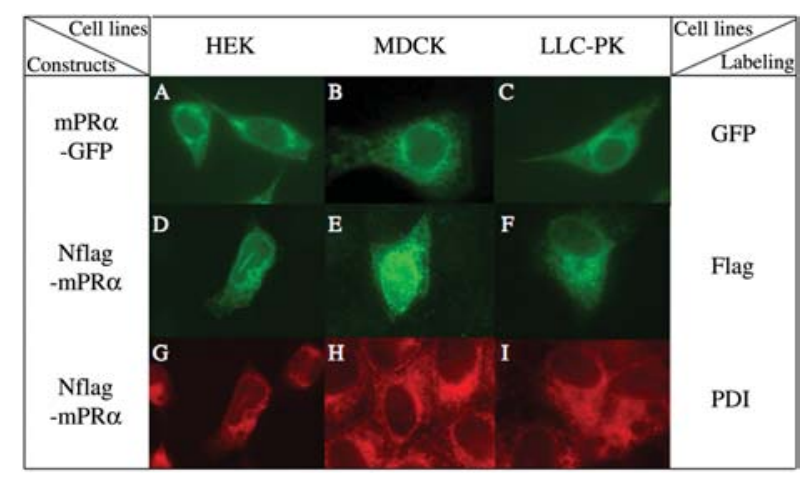

Figure 2 Murine $m P R \alpha$ localize to endoplasmic reticulum. HEK293 (A, D, G), MDCK (B, E, H), and LLC-PK (C, F, I) cell lines were transfected either GFP C-terminal fused $\mathrm{mPR} \alpha(\mathrm{A}-\mathrm{C})$ or N-terminal HA-tagged mPR $\alpha$ (D-I). Then, $24 \mathrm{~h}$ after transfection cells were fixed with $4 \%$ paraformaldehyde and directly observed under microscope or submitted to immunocytochemical analysis as described previously (30).

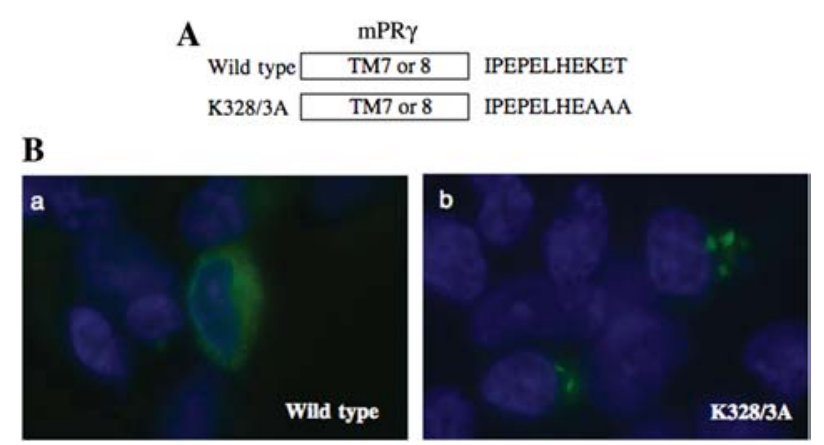

Figure 3 An endoplasmic reticulum retention motif in mouse $\mathrm{mPR} \gamma$.

(A) Schematic representation of the C-terminus tail from the last transmembrane domain (numbers 7 or 8 according to P. Thomas or T. Lyons model, respectively) showing the -KET motif replaced by three alanines in the mutant. (B) Wild type or K328/3A mutant were transfected in HEK293 for $24 \mathrm{~h}$. Cells were then treated as described previously (30).

sensus on the presence of mPRs into intracellular compartments (probably ER) owing to an intrinsic lysine-based retention motif indicating that this localization is programmed and not an artifact. The question is, therefore, to understand whether mPRs have to reach the cell surface to be active and what would be the processes, if they exist, that allow them to overcome the ER retention motif. An obvious possibility, recently outlined by Thomas et al. (25), is the absence of ER in which mPRs should be trapped as is the case in sperm cells. But beyond this particular case, is there a yet unidentified molecular mechanism involving posttranslational modifications of the receptor (phosphorylation, etc.) or association with a protein partner allowing maturation of mPRs to the cell surface? In some Western blot experiments, the Peter Thomas group observed an $80-\mathrm{kDa}$ band which was proposed to correspond to $\mathrm{mPR}$ dimers. Instead, it could represent a complex with another protein, which could explain the plasma membrane localization of mPRs. Research for putative partners might need to be performed to reconcile the different observations.

\section{mPRs, a new class of GPCRs?}

As mentioned above, identification of mPRs as GPCRs has raised major questions. Indeed, this assumption implies a seven-transmembrane domains topology with extracellular $\mathrm{N}$-terminus and intracellular $\mathrm{C}$-terminus. This predicted topology would suggest that all conserved motifs among PAQR family members are upside-down in mPR proteins when compared with the other members of this group. Homologous loops would be either intracellular or extracellular depending on the protein of the PAQR family they belong to. This situation is rather unusual as proteins of a same family generally not only share sequence homology but also have a common structural shape. For membrane-bound proteins belonging to the same family, a core structure formed by a group of transmembrane domains and intra- and extracellular loops is usually defined. This core structure can 
be "elongated" by additional transmembrane helixes. Phylogenetic analysis showed that PAQR family members, including $\mathrm{mPRs}$, have bacterial hemolysin as common ancestor, a gene restricted to Eubacteria (19). However, GPCR classes I, II, and III are related to bacterial rhodopsin, secretin, and glutamate-metabotropic receptors, respectively (37). The fact that mPRs, with this "Eubacterian" ancestor, would be GPCRs does not fit with established knowledge and would represent a completely new class of this gene family.

The seven-transmembrane model for mPR topology is based on mathematical predictions deduced from the amino acid sequence. Even if these predictions are good tools, they cannot replace experimental data. For instance, using a similar Kyte and Doolittle-based algorithm as Zhu et al. (14), other investigators $(33,34,38)$ proposed an eight-transmembrane domains topology for mPRs, which indicates that this in silico analysis is not reliable. These models, as suggested by Smith et al. (33), define a core structure common to all PAQR members composed of seven-transmembrane domains and predict the presence of an additional helix at the Cterminal side, in contradiction with the model suggested originally $(13,14)$.

The only experimental data partially resolving mPR topology focused on the localization of their $\mathrm{N}$ - and C-terminal extremities. As mentioned above, the Peter Thomas group $(21,22)$ reported data indicating that the $\mathrm{N}$-terminal part is extracellular because it is recognized by a specific antibody on non-permeabilized cells. By contrast, other reports using completely different strategies showed the presence of the Cterminal part in the cytoplasm $(30,33)$. It is not possible, to date, to draw final conclusions as the "real" topology of mPR owing to the lack of experimental data. This would necessitate a complete topological analysis that identifies all transmembrane domains, perhaps using a strategy involving fusion with a glycosylation reporter peptide cleverly placed within the mPR sequence.

Evidence for a $\mathrm{G}$ protein-induced pathway triggered by activation of mPRs has been totally refuted by Krietsch et al. (29). In this report, control experiments clearly established that this negative result was not related to a "technical" problem. A last result challenging the GPCR nature of mPRs comes from the data of Smith et al. (33), who reported that progesterone activates mPRs in yeast strains which do not express heterotrimeric $\mathrm{G}$ proteins. This group has proposed an alternative pathway involving a sphingoid base as a second messenger following activation of ceramidase enzymatic activity $(34,39)$. This transduction pathway needs to be tested in mammalian cells.

\section{Expert opinion}

The discovery of membrane-bound receptors specific for progesterone has opened an entirely new field of research. Only a few years after the identification of mPRs, a membrane-bound receptor for estrogen was concomitantly identified by two groups in 2005 (40, 41). Here again, debates regarding ligand-specificity and the cellular localization of this protein rapidly emerged and are still not resolved $(42,43)$. Accounting for the fact that rapid and non- genomic effects have been reported for the majority of steroids such as glucocorticosteroid (44), mineralocorticosteroid (45), or androgens $(46,47)$, the identification of mPRs opened the way for the discovery of other membrane-bound receptors for these molecules.

The purpose of this review was to summarize and confront the different data and points of view regarding the molecular characteristics of mPRs. By taking into account results that were confirmed by at least two independent groups, we can conclude that: (i) mPRs are progesterone receptors; (ii) under basal conditions or after transfection, mPRs are mainly localized into internal compartments, probably the ER; (iii) it is unlikely that mPRs are GPCRs; and (iv) their topology remains to be elucidated.

\section{Outlook}

Not surprisingly, a consensus regarding the rapid and nongenomic effects of progesterone mediated by mPRs has yet to emerge. The novelty of the observation and the sparse research groups fully involved in this area probably explain why it is not yet possible to draw the big picture of this phenomenon. Moreover, debates and controversies are part of the scientific process and are generally at the origin of new discoveries. It is because the classical action of steroids could not explain all observations that researchers explored other possibilities and discovered the different pathways which could explain rapid steroid effects. Thus, contradictions, non-reproducible data, or negative findings are not inherently problematic. However, we should not reach the point where these controversies are so important that they paralyze research in this domain by completely altering the central message. In this case, it would be impossible to attract new groups in this research field and to convince research organizations to financially support new studies.

In this review, we proposed a minimal consensus which seems acceptable today, based on observations that have been reproduced by several groups. New results in contradiction with this consensus could yet emerge. Efforts should be made to find a biological explanation for these putative discrepancies. Then, we could improve the "global dogma", regarding $\mathrm{mPR}$ characteristics, functions, and regulation. For instance, when we observed that mPRs were not translocated to the cell surface using "renal epithelial" cell lines, we searched for a "mechanistic" reason and found an ER retention/retrieval motif. Thus, the issue now is to understand in which situation(s) this motif can be either overcome to allow cell surface expression or "activated"' to retain mPR within the cells.

In our opinion, the data provided by the Lyons group have contributed to revive the research field that focused on mPRs. Indeed, by using a systematic approach in which mPRs were expressed in yeast (as other PAQR protein members), these authors have reported characteristics unaltered by possible regulative mechanisms. We can only encourage this systematic analysis where PAQR proteins are compared for their fundamental characteristics. 
The future of research on mPRs depends on the ability of leading groups to pass a "gentleman's agreement'" to give this area of research solid foundations, from which an understanding of the rapid and non-genomic action of progesterone, and more generally of steroids, will arise.

\section{Acknowledgments}

We are grateful to Dr A. Edwards (Tufts University, MA, USA) for her helpful corrections of the manuscript. This study was supported by grants from the Centre National de Recherche Scientifique (CNRS) via its Action Thématique et Incitative sur Programme (ATIP grant) (G.C.), from Amgen (G.C.). A.S. is a PhD student funded by the French Society of Nephrology.

A.S., J.L., N.P., M.B.F., and G.C. have no conflict of interest to disclose.

\section{References}

1. Selye H. Correlation between the chemical structure and the pharmacological actions of steroids. Endocrinology 1942; 30: 437-53.

2. Szego CM, Davis JS. Adenosine $3^{\prime}, 5^{\prime}$-monophosphate in rat uterus: acute elevation by estrogen. Proc Natl Acad Sci USA 1967; 58: 1711-8.

3. Norman AW, Mizwicki MT, Norman DP. Steroid-hormone rapid actions, membrane receptors and a conformational ensemble model. Nat Rev Drug Discov 2004; 3: 27-41.

4. Kostellow AB, Ziegler D, Morrill GA. Regulation of $\mathrm{Ca}^{2+}$ and cyclic AMP during the first meiotic division in amphibian oocytes by progesterone. J Cyclic Nucleotide Res 1980; 6: 347-58.

5. Baldi E, Krausz C, Forti G. Nongenomic actions of progesterone on human spermatozoa. Trends Endocrinol Metab 1995; 6: 198-205.

6. Brunette MG, Leclerc M. Renal action of progesterone: effect on calcium reabsorption. Mol Cell Endocrinol 2002; 194: 183-90.

7. Brunette MG, Leclerc M. Effect of estrogen on calcium and sodium transport by the nephron luminal membranes. J Endocrinol 2001; 170: 441-50.

8. Cato AC, Nestl A, Mink S. Rapid actions of steroid receptors in cellular signaling pathways. Sci STKE 2002; 138: re9.

9. Fernandes MS, Brosens JJ, Gellersen B. Honey, we need to talk about the membrane progestin receptors. Steroids 2008; 73: 942-52.

10. Trant JM, Thomas P, Shackleton $\mathrm{CH}$. Identification of 17 alpha,20 beta,21-trihydroxy-4-pregnen-3-one as the major ovarian steroid produced by the teleost Micropogonias undulatus during final oocyte maturation. Steroids 1986; 47: 89-99.

11. Trant JM, Thomas P. Isolation of a novel maturation-inducing steroid produced in vitro by ovaries of Atlantic croaker. Gen Comp Endocrinol 1989; 75: 397-404.

12. Patino R, Thomas P. Characterization of membrane receptor activity for 17 alpha, 20 beta, 21-trihydroxy-4-pregnen-3-one in ovaries of spotted seatrout (Cynoscion nebulosus). Gen Comp Endocrinol 1990; 78: 204-17.

13. Zhu Y, Rice CD, Pang Y, Pace M, Thomas P. Cloning, expression, and characterization of a membrane progestin receptor and evidence it is an intermediary in meiotic maturation of fish oocytes. Proc Natl Acad Sci USA 2003; 100: 2231-6.
14. Zhu Y, Bond J, Thomas P. Identification, classification, and partial characterization of genes in humans and other vertebrates homologous to a fish membrane progestin receptor. Proc Natl Acad Sci USA 2003; 100: 2237-42.

15. Grimont A, Bloch-Faure M, El Abida B, Crambert G. Mapping of sex hormone receptors and their modulators along the nephron of male and female mice. FEBS Lett 2009; 583: 1644-8.

16. Yamauchi T, Kamon J, Ito Y, Tsuchida A, Yokomizo T, Kita S, Sugiyama T, Miyagishi M, Hara K, Tsunoda M, Murakami K, Ohteki T, Uchida S, Takekawa S, Waki H, Tsuno NH, Shibata Y, Terauchi Y, Froguel P, Tobe K, Koyasu S, Taira K, Kitamura T, Shimizu T, Nagai R, Kadowaki T. Cloning of adiponectin receptors that mediate antidiabetic metabolic effects. Nature 2003; 423: 762-9.

17. Narasimhan ML, Coca MA, Jin J, Yamauchi T, Ito Y, Kadowaki T, Kim KK, Pardo JM, Damsz B, Hasegawa PM, Yun DJ, Bressan RA. Osmotin is a homolog of mammalian adiponectin and controls apoptosis in yeast through a homolog of mammalian adiponectin receptor. Mol Cell 2005; 17: 171-80.

18. Luo X, Feng L, Jiang X, Xiao F, Wang Z, Feng GS, Chen Y. Characterization of the topology and functional domains of RKTG. Biochem J 2008; 414: 399-406.

19. Tang YT, Hu T, Arterburn M, Boyle B, Bright JM, Emtage PC, Funk WD. PAQR proteins: a novel membrane receptor family defined by an ancient 7-transmembrane pass motif. J Mol Evol 2005; 61: 372-80.

20. Hsieh MH, Goodman HM. A novel gene family in Arabidopsis encoding putative heptahelical transmembrane proteins homologous to human adiponectin receptors and progestin receptors. J Exp Bot 2005; 56: 3137-47.

21. Hanna R, Pang Y, Thomas P, Zhu Y. Cell-surface expression, progestin binding, and rapid nongenomic signaling of zebrafish membrane progestin receptors alpha and beta in transfected cells. J Endocrinol 2006; 190: 247-60.

22. Thomas P, Pang Y, Dong J, Groenen P, Kelder J, de Vlieg J, Zhu Y, Tubbs C. Steroid and G protein binding characteristics of the seatrout and human progestin membrane receptor alpha subtypes and their evolutionary origins. Endocrinology 2007; 148: 705-18.

23. Karteris E, Zervou S, Pang Y, Dong J, Hillhouse EW, Randeva HS, Thomas P. Progesterone signaling in human myometrium through two novel membrane $G$ protein-coupled receptors: potential role in functional progesterone withdrawal at term. Mol Endocrinol 2006; 20: 1519-34.

24. Tubbs C, Thomas P. Progestin signaling through an olfactory $\mathrm{G}$ protein and membrane progestin receptor-alpha in Atlantic croaker sperm: potential role in induction of sperm hypermotility. Endocrinology 2009; 150: 473-84.

25. Thomas P, Tubbs C, Garry VF. Progestin functions in vertebrate gametes mediated by membrane progestin receptors (mPRs): identification of mPRalpha on human sperm and its association with sperm motility. Steroids 2009; 74: 614-21.

26. Zhu Y, Hanna RN, Schaaf MJ, Spaink HP, Thomas P. Candidates for membrane progestin receptors - past approaches and future challenges. Comp Biochem Physiol C Toxicol Pharmacol 2008; 148: 381-9.

27. Dressing GE, Thomas P. Identification of membrane progestin receptors in human breast cancer cell lines and biopsies and their potential involvement in breast cancer. Steroids 2007; 72: 111-6.

28. Thomas P, Tubbs C, Detweiler C, Das S, Ford L, BreckenridgeMiller D. Binding characteristics, hormonal regulation and 
identity of the sperm membrane progestin receptor in Atlantic croaker. Steroids 2005; 70: 427-33.

29. Krietsch T, Fernandes MS, Kero J, Losel R, Heyens M, Lam EW, Huhtaniemi I, Brosens JJ, Gellersen B. Human homologs of the putative $\mathrm{G}$ protein-coupled membrane progestin receptors (mPRalpha, beta, and gamma) localize to the endoplasmic reticulum and are not activated by progesterone. Mol Endocrinol 2006; 20: 3146-64.

30. Lemale J, Bloch-Faure M, Grimont A, El Abida B, ImbertTeboul M, Crambert G. Membrane progestin receptors alpha and gamma in renal epithelium. Biochim Biophys Acta 2008; 1783: 2234-40.

31. Ashley RL, Arreguin-Arevalo JA, Nett TM. Binding characteristics of the ovine membrane progesterone receptor alpha and expression of the receptor during the estrous cycle. Reprod Biol Endocrinol 2009; 7: 42-51.

32. Kupchak BR, Garitaonandia I, Villa NY, Mullen MB, Weaver MG, Regalla LM, Kendall EA, Lyons TJ. Probing the mechanism of FET3 repression by Izh2p overexpression. Biochim Biophys Acta 2007; 1773: 1124-32.

33. Smith JL, Kupchak BR, Garitaonandia I, Hoang LK, Maina AS, Regalla LM, Lyons TJ. Heterologous expression of human mPRalpha, mPRbeta and mPRgamma in yeast confirms their ability to function as membrane progesterone receptors. Steroids 2008; 73: 1160-73.

34. Kupchak BR, Garitaonandia I, Villa NY, Smith JL, Lyons TJ. Antagonism of human adiponectin receptors and their membrane progesterone receptor paralogs by TNFalpha and a ceramidase inhibitor. Biochemistry 2009; 48: 5504-6.

35. Ashley RL, Clay CM, Farmerie TA, Niswender GD, Nett TM. Cloning and characterization of an ovine intracellular seven transmembrane receptor for progesterone that mediates calcium mobilization. Endocrinology 2006; 147: 4151-9.

36. Fernandes MS, Pierron V, Michalovich D, Astle S, Thornton S, Peltoketo H, Lam EW, Gellersen B, Huhtaniemi I, Allen J, Brosens JJ. Regulated expression of putative membrane progestin receptor homologues in human endometrium and gestational tissues. J Endocrinol 2005;187: 89-101.

37. Pierce KL, Premont RT, Lefkowitz RJ. Seven-transmembrane receptors. Nat Rev Mol Cell Biol 2002; 3: 639-50.
38. Mourot B, Nguyen T, Fostier A, Bobe J. Two unrelated putative membrane-bound progestin receptors, progesterone membrane receptor component 1 (PGMRC1) and membrane progestin receptor (mPR) beta, are expressed in the rainbow trout oocyte and exhibit similar ovarian expression patterns. Reprod Biol Endocrinol 2006; 4: 6.

39. Villa NY, Kupchak BR, Garitaonandia I, Smith JL, Alonso E, Alford C, Cowart LA, Hannun YA, Lyons TJ. Sphingolipids function as downstream effectors of a fungal PAQR. Mol Pharmacol 2009; 75: 866-75.

40. Thomas P, Pang Y, Filardo EJ, Dong J. Identity of an estrogen membrane receptor coupled to a $G$ protein in human breast cancer cells. Endocrinology 2005; 146: 624-32.

41. Revankar CM, Cimino DF, Sklar LA, Arterburn JB, Prossnitz ER. A transmembrane intracellular estrogen receptor mediates rapid cell signaling. Science 2005; 307: 1625-30.

42. Maggiolini M, Picard D. The unfolding stories of GPR30, a new membrane-bound estrogen receptor. J Endocrinol 2010; 204: 105-14.

43. Otto C, Rohde-Schulz B, Schwarz G, Fuchs I, Klewer M, Brittain D, Langer G, Bader B, Prelle K, Nubbemeyer R, Fritzemeier KH. G protein-coupled receptor 30 localizes to the endoplasmic reticulum and is not activated by estradiol. Endocrinology 2008; 149: 4846-56.

44. Tasker JG, Di S, Malcher-Lopes R. Minireview: rapid glucocorticoid signaling via membrane-associated receptors. Endocrinology 2006; 147: 5549-56.

45. Le Moellic C, Ouvrard-Pascaud A, Capurro C, Cluzeaud F, Fay M, Jaisser F, Farman N, Blot-Chabaud M. Early nongenomic events in aldosterone action in renal collecting duct cells: PKCalpha activation, mineralocorticoid receptor phosphorylation, and cross-talk with the genomic response. J Am Soc Nephrol 2004; 15: 1145-60.

46. Benten WP, Guo Z, Krucken J, Wunderlich F. Rapid effects of androgens in macrophages. Steroids 2004; 69: 585-90.

47. Lieberherr M, Grosse B. Androgens increase intracellular calcium concentration and inositol 1,4,5-trisphosphate and diacylglycerol formation via a pertussis toxin-sensitive G-protein. J Biol Chem 1994; 269: 7217-23. 